\title{
Prediction of MicroRNAs Bind to Toll-like Receptors Pathway in Chicken Based on Bioinformatic Methods
}

\author{
Maryam Gholizadeh $^{1}$ (D, Jamal Fayazi ${ }^{*}$ (D), Somayeh Rahimnahal ${ }^{1}$ (D)
}

1. Department of Animal Science, Faculty of Animal and Food Science, Agricultural Sciences and Natural Resources University of Khuzestan, Ahvaz, Iran.

\begin{tabular}{l|l}
$\begin{array}{c}\text { Use yur devic to scan } \\
\text { and read the article online }\end{array}$ \\
Based on Bioinformatic Methods. Research in Molecular Medicine. 2019; 7(4):33-42. https://doi.org/10.32598/rmm.7.4.33
\end{tabular}

\section{(i) (\$)}

Article Type:

Research Article

Article info:

Received: 19 Sep 2019

Revised: 12 Oct 2019

Accepted: 29 Oct 2019

Keywords:

Toll-like receptors pathway, microRNAs, Bioinformatic method, Inflammatory diseases

\begin{abstract}
A B S T RA C T
Background: Toll-like receptors (TLRs) detect diverse pathogen-associated molecular patterns and play a critical role in the innate immune response. Hosts should activate TLR-signaling pathways to eliminate invading pathogens. However, excessive activation of these pathways may interrupt immune homeostasis, leading to several diseases. Therefore precise regulation of TLR-signaling pathways is essential. Meanwhile, miRNAs (microRNAs) act similar to a class of small noncoding RNAs with gene regulatory functions. The regulation of TLR expression by miRNAs may be one of the valid points for targeting TLRs.

Materials and Methods: In this study, we predicted most of the microRNAs bind to the TLRs pathway in the chicken, based on the bioinformatic methods. All genes involved in the TLR signaling pathway in chicken species were extracted from the KEGG database (Entry: gga04620) and analyzed based on different applications.

Results: We predicted 19 miRNAs for the 18 target genes of the TLR pathway that may provide essential clues for identifying novel drug targets for inflammatory diseases.

Conclusion: Substantial miRNA was found as gene regulators. As newly identified regulators, the performance mechanism of miRNAs in combination with other regulatory mechanisms will control the outcome of immune responses and these issues should be investigated in future studies
\end{abstract}

\section{Introduction}

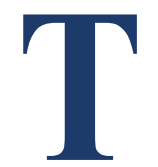

oll-Like Receptors (TLRs) are members of a large family of Pattern Recognition Receptors (PRRs) system, responsible for the recognition of Pathogen-Associated Molecular Patterns (PAMPs) of infectious pathogens [1]. Following the PAMPs recognition, PRRs initiate innate immune responses by activating intracellular signaling cascades that lead to a transcriptional expression of inflammatory mediators. Studies of the TLR signaling pathway are most advanced in mouse and human [2]; however, research is limited to a series of TLRs in other vertebrates, including fish, chickens, cattle, sheep, dog, and pig [1, 3-5].

\footnotetext{
* Corresponding Author:
}

Jamal Fayazi, PhD.

Address: Department of Animal Science, Faculty of Animal and Food Science, Agricultural Sciences and Natural Resources University of Khuzestan, Ahvaz, Iran.

Phone: +98 (916) 6124162

E-mail:j_fayazi@asnrukh.ac.ir 
Key features of this pathway are similar between birds and mammals, but some definite differences exist, too. Chicken TLR collection consists of 10 genes similar to humans, and TLRs 3, 4, 5, and 7 are orthologous in other vertebrates [6]. TLRs signaling pathway is regulated by positive or negative conflation at multiple levels to prevent excessive inflammation. Several mechanisms and molecules are responsible for the regulation of the TLR-signaling pathways. Among many regulatory molecules, microRNAs (miRNAs) have received extensive consideration as a newly identified family of regulators involved in fine-tuning of TLR-signaling pathways.

These miRNAs are small single-stranded noncoding RNAs (approximately 19 to 24 nucleotides long) with the significant role of regulating gene expression in developmental processes. Many studies have demonstrated that genes expression profiles of TLR-signaling pathways are subject to change when controlled by annealing to miRNAs. The first study that indicated the regulatory role of miRNAs in the immune response published in 2004 with a report showed selective expression of miR142a, miR-181a, and miR-223 in immune cells 14 .

Baltimore et al. demonstrated that the upregulating expression of miR-132, miR-155, and miR-146a is associated with the stimulation of lipopolysaccharide [7]. Quinn et al. investigated on a trio of miRNAs (miR-155, miR-21, and miR-146) with proven-control over TLR signaling and many immune and inflammatory pathways [5]. Using the bioinformatic methods, Cui et al. predicted the role of miR-8159 that was explored in regulating TLR13 and was involved in inflammatory responses of miiuy croaker [1]. Since the miRNAs may serve as significant regulators for adjusting the differentiation of immune cells as well as the immune responses to pathogens, this study provides the basic idea to detect miRNA in chicken that regulates gene expressions of TLR-signaling pathways based on bioinformatic methods.

\section{Materials and Methods}

All genes involved in the TLR-signaling pathway in chicken species were extracted from the KEGG database (KEGG database pathway: gga04620) and examined for this study. A large number of tools for miRNA prediction have been developed in recent years based on computational algorithms. These tools identify miRNAs using features such as sequence conservation, structure, thermodynamic parameters, and sequence-specific parameters. miRDB is an online database for miRNA target prediction and functional annotations (http://mirdb.org/ miRDB/index.html) [8].
All targets in miRDB are predicted by a bioinformatics tool, MirTarget [9], which has been developed by examining thousands of miRNA-target interactions from high-throughput sequencing experiments. Common features related to miRNA target binding have been identified and used to predict miRNA targets with machine learning methods. miRDB hosts have predicted miRNA targets in five species: human, mouse, rat, dog, and chicken. For the initial stage for the prediction of miRNA, the total genes of the TLR-signaling pathway were analyzed by miRDB software. Information includes total miRNA prediction, gene symbol, NCBI GeneID, 3'-UTR length, 3'-UTR sequence, miRNA name, target score, and miRNA sequence.

The seed location for each gene was obtained with miRDB software. In the second stage, the outputs of miRDB with high scores were analyzed by miRmap. It is an open-source software library that integrates thermodynamic, evolutionary, probabilistic, and sequencebased features. Thermodynamic features of miRmap include " $\Delta \mathrm{G}$ duplex" and " $\Delta \mathrm{G}$ binding" that assess the energy of the miRNA-mRNA duplex, " $\Delta \mathrm{G}$ open" that evaluates the energy necessary to unfold the 3'-UTR and make the area accessible for RISC binding and " $\Delta \mathrm{G}$ total" (also named ' $\Delta \Delta G$ ' by Kertesz et al. [10]) that sums " $\Delta \mathrm{G}$ duplex" and " $\Delta \mathrm{G}$ open". Since the publication of the miRmap library, we have added new features, i.e. " $\Delta \mathrm{G}$ seed duplex" and " $\Delta \mathrm{G}$ seed binding", that specifically evaluates the seed region.

miRmap allows us to examine feature correlations and to compare their predictive power in an unbiased way using high throughput experimental data from immune purification, transcriptomics, proteomics, and polysome fractionation experiments [6]. This software is used for the prediction of miRNAs in human, mouse, rat, chicken, and other metazoan genomes. The output of miRmap software for each gene includes gene name, miRNA name, miRmap score, $\Delta \mathrm{G}$ duplex, $\Delta \mathrm{G}$ open, and multiple binding sites.

\section{Results}

Genes involved in the TLR signaling pathway in chicken

Gene list of chicken TLR-signaling pathways containing 83 genes were extracted from the KEGG database that (Table 1). Different TLRs genes play crucial roles in the activation of the immune response to PAMPs. TLR1, TLR2, TLR4, TLR5 tend to specialize in recognition of bacterial components present on the outer surfaces, 
Table 1. Some of 83 genes List in chicken TLR signaling pathway

\begin{tabular}{|c|c|c|c|c|c|c|c|}
\hline Gene Symbol & GenelD & Gene Symbol & GenelD & Gene Symbol & GenelD & Gene Symbol & GenelD \\
\hline TLR1A & 426274 & LOC107050551 & 107050551 & CHUK & 423669 & PIK3R2 & 771142 \\
\hline TLR1B & 771173 & MAPK11 & 417739 & IKBKB & 426792 & PIK3R1 & 427171 \\
\hline TLR2A & 374141 & MAPK14 & 421183 & IKBKB & 107054958 & AKT1 & 395928 \\
\hline TLR2B & 769014 & MAPK12 & 769763 & NFKBIA & 396093 & AKT3 & 421497 \\
\hline TLR2L & 100858824 & MAPK13 & 771145 & NFKB1 & 396033 & TOLLIP & 423099 \\
\hline LY96 & 420189 & MAPK8 & 423778 & RELA & 396027 & MYD88 & 420420 \\
\hline TLR3 & 422720 & MAРК9 & 395983 & МАР3К8 & 420479 & TIRAP & 419715 \\
\hline TLR4 & 417241 & MAPK10 & 422592 & MAP2K1 & 415549 & FADD & 423146 \\
\hline TLR5 & 554217 & JUN & 424673 & MAP2K2 & 396349 & CASP8 & 395284 \\
\hline TLR7 & 418638 & FOS & 396512 & MAPK1 & 373953 & CASP8L & 107056614 \\
\hline CTSK & 395818 & IL1B & 395196 & MAP2K3 & 416496 & IRAK4 & 417796 \\
\hline RAC1 & 395871 & IL6 & 395337 & MAP2K6 & 417445 & FNA3 & 396398 \\
\hline PIK3CA & 424971 & IL12A & 407090 & MAP2K4 & 417312 & CD86 & 427944 \\
\hline PIK3CD & 419444 & IL12B & 404671 & LOC100859032 & 100859032 & IRF5 & 430409 \\
\hline PIK3СB & 424826 & IL8L2 & 396495 & LOC100858177 & 100858177 & IRF7 & 396330 \\
\hline PIK3R3 & 429096 & IL8L1 & 395872 & LOC100857947 & 100857947 & SPP1 & 395210 \\
\hline TRAF6 & 423163 & CCL5 & 417465 & LOC100857744 & 100857744 & IKBKE & 430480 \\
\hline TAB1 & 418014 & CCL4 & 395551 & IFNKL & 101750245 & TBK1 & 417825 \\
\hline TAB2 & 421622 & TICAM1 & 100008585 & LOC107049337 & 107049337 & TRAF3 & 423471 \\
\hline МАР3К7 & 421808 & RIPK1 & 378921 & IFNW1 & 554219 & CD40 & 395385 \\
\hline CD80 & 768950 & STAT1 & 424044 & IFNAR1 & 395665 & IFA3L & 768614 \\
\hline IFNAR2 & 395664 & - & - & - & - & - & - \\
\hline
\end{tabular}

whereas TLR3 and TLR7 specialize in recognizing nucleic acids, especially of viral origin. In the chicken, before the genome release, in silico clustering of expressed sequence tags revealed homologs of the TLR pathway, including two TLRs, one homologous to human TLR3 and the other similar to human and mouse TLR1, TLR6, and TLR10 $[3,11]$.

Other chicken TLR-pathway genes identified include those encoding Toll Interacting Protein (TOLLIP), Interleukin-1 Receptor-Associated Kinase 4 (IRAK4), Myeloid Differentiation factor 88 (MyD88), MyD88adapter-like protein (Mal or TIRAP), Tak1-binding proteins 1 and 2 (TAB1 and TAB2), Tumor Necrosis Factor
Receptor (TNFR)-Associated Factor 6 (TRAF6), Transforming Growth Factor-beta (TGF)-Activated Kinase (TAK1), and IB kinases and IKK $[9,12]$.

\section{Properties of Chicken TLR}

The chicken TLR repertoire consists of 10 genes (Table 2). Phylogenetic analyses show that 6 genes have orthologues in mammals and fish, while one is only shared by fish, and three appear to be unique to birds. In the chicken, TLR2 and TLR4 have been identified and characterized at molecular and functional levels [6]. Comparisons of structural predictions for the chicken TLRs 
Table 2. List of chicken TLR genes

\begin{tabular}{cccccccc}
\hline Gene symbol & Chromosome & Location & $\begin{array}{c}\text { Number } \\
\text { of Exons }\end{array}$ & $\begin{array}{c}\text { Length } \\
\text { (aa) }\end{array}$ & $\begin{array}{c}\text { Length } \\
\text { (genomic) }\end{array}$ & $\begin{array}{c}\text { Genebank } \\
\text { Nucleotide }\end{array}$ & Genebank Protein \\
\hline TLR1LA & 4 & $41563594-71566050$ & 1 & 818 & 2457 & AB109401 & BAD67422 \\
\hline TLR1LB & 4 & $71553122-71555080$ & 1 & 722 & 5005 & DQ518918 & ABF67957 \\
\hline TLR2A & 4 & $21105675-21108056$ & 1 & 793 & 2382 & NM204278 & NP989609 \\
\hline TLR2B & 4 & $21113342-21115936$ & 1 & 781 & 2594 & AB046533 & Q9DGB6 \\
\hline TLR3 & 4 & $63155888-63160902$ & 4 & 896 & 5015 & NM001011691 & NP_001011691 \\
\hline TLR4 & 17 & $4062994-4067445$ & 3 & 843 & 4452 & AY064697 & AAL49971 \\
\hline TLR5 & 3 & $18975945-18978530$ & 1 & 862 & 2589 & AJ626848 & CAF25167 \\
\hline TLR7 & 1 & $126824071-126830542$ & 2 & 1059 & 6381 & AJ626848 & CAF25167 \\
\hline TLR15 & 3 & $2945856-2948462$ & 1 & 868 & 2607 & XM419294 & XP419294 \\
\hline TLR21 & 11 & $338885-342202$ & 2 & 972 & 3317 & NM001030558 & NP001025729 \\
\hline
\end{tabular}

shown they have a pattern of gene duplication and gene loss when compared to mammals.

Fukui et al. identified two types of chicken TLR2 that both forms (TLR2A and TLR2B), with identical TIR domains, have been mapped to the same region of chromosome 4 and suggested that they arose by gene duplication [12]. Chicken Expressed Sequence Tag (ESTs) have been identified with the highest homology to mammalian TLR1, TLR6, or TLR10, and TLR3 [9].

All TLRs except TLR3 can signal through the adaptor molecule MyD 88 (myeloid differentiation factor 88 ). In the MyD 88-dependent pathway, the adaptor is bound to TLR's intracellular domain. The newly discovered TLR15 has the unusual feature of many LRRs clustered towards the $\mathrm{C}$-terminus of the molecule and few at the N-terminus. TLR7 has the uncharacteristic feature of a splice variant with two predicted transmembrane domains only previously found in mammalian.

Result of miRNA target search with the standard query interface in miRDB

miRNA regulates gene expression mainly by annealing to a complementary region within the 3 '-UTR of their target mRNA. The result of miRDB showed that of 83 genes involved in the TLR pathway, only 70 genes were detected as miRNA targets. Other genes were not the targets of miRNAs recorded in the miRNA database for Gallus species. A gene can have some binding sites with one or several miRNAs [13]. The output of software re- ported annealing to a target in 3'-UTR with score $>50$, so 1119 miRNAs were detected for 70 genes. To enhance the accuracy and prevent the creation of false-positive errors, we considered annealing with a target score $>80$ for the next analysis, so 247 miRNAs were confirmed for target genes.

In this software, major factors were searched for prediction miRNA to find a perfect complementary seed. The seed length for this program is between 6 and 8 nucleotides that shorter length leads to the prediction of the considerable quantity of false sites. AKT3 gene has 3'-UTR length of 3565 and 53 binding sites for 38 miRNA that have most miRNA binding sites among the 70 genes of the TLR pathway ( 6 connection with target score $=100$ ).

Positions 12, 206, 1058, and 3133 in the 3-UTR of AKT3 gene with similar seed sequence were targets for several miRNAs, including gga-miR-15a, gga-miR-165p, gga-miR-16c-5p, gga-miR-15b-5p, and gga-miR15c-5p (seed sequence: AGCAGC and 3-UTR: TGCTGCT). Positions 205, 2563, and 3132 were targets for gga-miR-6598-5p that sequence TTGCTGC of 3-UTR annealing with seed sequence UGCAGC. NFKB1, MAP2K2, MAPK13, IL6, IRF7, TRAF3, and IFNAR1 have only one binding site for miRNA.

\section{Comprehensive microRNA target prediction}

Using the 3-UTR sequence and miRNA, miRDB predicted all genes (70 genes were targets for $247 \mathrm{miR}$ - 
Table 3. Results of miRmap analysis confirming only 27 genes and 67 miRNAs out of 70 genes and 247 miRNAs that were as input

\begin{tabular}{|c|c|c|c|c|c|}
\hline Gene Symbol & miRNA Name & miRmap Score & $\Delta \mathrm{G}$ Duplex & $\Delta \mathrm{G}$ Open & Binding Sites \\
\hline LOC100858824 & gga-miR-15a & 0.35 & -12.07 & 7.07 & 8 \\
\hline LOC100858824 & gga-miR-16-5p & 0.39 & -12.15 & 8.37 & 8 \\
\hline LOC100858824 & gga-miR-15c-5p & 0.36 & -12.83 & 8.37 & 8 \\
\hline LOC100858824 & gga-miR-15b-5p & 0.35 & -13.2 & 8.37 & 8 \\
\hline LOC100858824 & gga-miR-16c-5p & 0.36 & -12.72 & 8.37 & 8 \\
\hline PIK3CD & gga-miR-196-1-3p & 0.48 & -7.36 & 8.30 & 3 \\
\hline РІК3СВ & gga-miR-214 & 0.10 & -15.02 & 13.72 & 4 \\
\hline РІКЗСВ & gga-miR-21-3p & 0.04 & -12.05 & 8.65 & 2 \\
\hline PIK3R5 & gga-miR-6609-3p & 0.08 & -19.55 & 21.27 & 2 \\
\hline PIK3R2 & gga-miR-3536 & 0.00 & -13.70 & 33.56 & 1 \\
\hline PIK3R1 & gga-miR-383-3p & 0.43 & -13.08 & 12.73 & 5 \\
\hline PIK3R1 & gga-miR-6544-5p & 0.86 & -8.45 & 14.72 & 2 \\
\hline PIK3R1 & gga-miR-6550-5p & 0.62 & -12.96 & 8.47 & 8 \\
\hline PIK3R1 & gga-miR-6699-5p & 0.87 & -11.35 & 8.21 & 7 \\
\hline PIK3R1 & gga-miR-1617 & 0.13 & -16.73 & 13.33 & 3 \\
\hline PIK3R1 & gga-miR-1687-5p & 0.64 & -10.48 & 2.18 & 7 \\
\hline АКT3 & gga-miR-15c-5p & -0.36 & -16.00 & 9.09 & 5 \\
\hline AKT3 & gga-miR-15b-5p & -0.39 & -14.28 & 9.09 & 6 \\
\hline AKT3 & gga-miR-16c-5p & -0.36 & -16.05 & 9.09 & 6 \\
\hline AKT3 & gga-miR-16-5p & -0.35 & -14.13 & 9.09 & 6 \\
\hline АКT3 & gga-miR-6598-5p & 0.11 & 14.84 & 7.20 & 7 \\
\hline АKT3 & gga-miR-15a & -0.35 & -12.85 & 9.05 & 6 \\
\hline АКT3 & gga-miR-1612 & 4.01 & -7.82 & 6.04 & 21 \\
\hline TOLLIP & gga-miR-383-3p & -0.08 & -16.95 & 13.39 & 2 \\
\hline TOLLIP & gga-miR-16-5p & -0.13 & -12.45 & 8.84 & 7 \\
\hline TOLLIP & gga-miR-15c-5p & -0.14 & -12.01 & 8.84 & 7 \\
\hline TOLLIP & gga-miR-15b-5p & -0.14 & -12.20 & 8.84 & 7 \\
\hline TOLLIP & gga-miR-15a & -0.12 & -11.59 & 7.63 & 7 \\
\hline TOLLIP & gga-miR-7456-3p & 0.18 & -11.8 & 7.57 & 5 \\
\hline TOLLIP & gga-miR-6706-5p & 0.64 & -12.42 & 10.16 & 5 \\
\hline MYD88 & gga-miR-7442-5p & 0.38 & -15.52 & 9.06 & 5 \\
\hline MYD88 & gga-miR-1804 & 0.30 & -14.94 & 13.11 & 5 \\
\hline FADD & gga-miR-1612 & 5.58 & -7.67 & 3.66 & 29 \\
\hline TRAF6 & gga-miR-6562-5p & 0.27 & -15.23 & 9.70 & 6 \\
\hline TAB1 & gga-miR-3594-5p & 0.12 & -16.73 & 10.55 & 2 \\
\hline TAB1 & gga-miR-1623 & -0.06 & -18.55 & 15.51 & 2 \\
\hline TAB2 & gga-miR-1704 & 0.05 & -15.96 & 12.39 & 3 \\
\hline TAB2 & gga-miR-1625-3p & 0.24 & -14.50 & 12.58 & 3 \\
\hline TAB2 & gga-miR-6598-5p & -0.01 & -12.50 & 12.75 & 1 \\
\hline MAP3K7 & gga-miR-9-3p & 0.24 & -12.45 & 13.00 & 2 \\
\hline MAP3K7 & gga-miR-1730-5p & 0.01 & -16.10 & 22.81 & 1 \\
\hline MAP2K2 & gga-miR-1757 & -0.25 & -18.01 & 10.51 & 2 \\
\hline MAP2K3 & gga-miR-1754-3p & -1.00 & -19.35 & 17.97 & 10 \\
\hline MAP2K3 & gga-miR-6609-3p & -0.87 & -21.74 & 18.22 & 8 \\
\hline
\end{tabular}




\begin{tabular}{|c|c|c|c|c|c|}
\hline Gene Symbol & miRNA Name & miRmap Score & $\Delta \mathrm{G}$ Duplex & $\Delta G$ Open & Binding Sites \\
\hline MAP2K6 & gga-miR-1590 & -0.09 & -15.10 & 9.99 & 1 \\
\hline MAP2K6 & gga-miR-1574-3p & -0.13 & -15.00 & 7.56 & 2 \\
\hline MAPK14 & gga-miR-6639-5p & 0.21 & -13.07 & 11.50 & 6 \\
\hline MAPK13 & gga-miR-7456-3p & 0.04 & -16.36 & 6.10 & 15 \\
\hline MAPK13 & gga-miR-1599 & 0.18 & -14.78 & 5.77 & 10 \\
\hline MAPK8 & gga-miR-15a & -0.31 & -17.12 & 11.12 & 2 \\
\hline MAPK8 & gga-miR-15c-5p & -0.30 & 15.55 & 11.11 & 2 \\
\hline MAPK8 & gga-miR-16c-5p & -0.29 & -15.00 & 11.11 & 2 \\
\hline MAPK8 & gga-miR-196-2-3p & -0.30 & -16.02 & 11.11 & 2 \\
\hline MAPK8 & gga-miR-15b-5p & -0.33 & -15.21 & 11.11 & 2 \\
\hline MAPK8 & gga-miR-107-3p & 0.04 & -16.30 & 11.08 & 2 \\
\hline MAPK8 & gga-miR-103-3p & 0.04 & -16.30 & 11.08 & 2 \\
\hline MAPK9 & gga-miR-6667-5p & $0.13-$ & -16.20 & 2.16 & 1 \\
\hline MAPK9 & gga-miR-6566-3p & -0.12 & -15.08 & 12.60 & 2 \\
\hline MAPK10 & gga-miR-6562-5p & 0.23 & -14.12 & 7.29 & 13 \\
\hline RIPK1 & gga-miR-6633-5p & 0.25 & -11.85 & 14.17 & 2 \\
\hline RIPK1 & gga-miR-1687-5p & 0.26 & -10.40 & 13.78 & 2 \\
\hline TBK1 & gga-miR-1687-5p & 0.42 & -12.95 & 12.84 & 4 \\
\hline TBK1 & gga-miR-6633-5p & 0.61 & -13.08 & 13.11 & 5 \\
\hline STAT1 & gga-miR-30c-1-3p & -1.15 & -14.11 & 1.15 & 12 \\
\hline STAT1 & gga-miR-1599 & -1.18 & -14.05 & 1.18 & 12 \\
\hline IRF7 & gga-miR-1723 & -0.21 & -20.80 & 31.94 & 1 \\
\hline IFNAR1 & gga-miR-1768 & -0.11 & -14.60 & 22.76 & 1 \\
\hline
\end{tabular}

NAs), then this sequence is analyzed one by one with miRmap algorithm to find miRNAs that was approved in both applications. The result of miRmap analysis was confirmed only 27 genes and 67 miRNAs of 70 genes and 247 miRNAs that were as input software (Table 3).

FADD gene contains 29 binding sites for ggamiR-1612 with a target score of 5.8; energy-duplex, -6.77 , and $\Delta \mathrm{G}$ open, 3.66 , which was the highest score and number of binding sites between 70 genes. Analysis of this gene with miRDB represents 10 miRNA binding sites for 4 miRNAs that 2 binding sites belonged to gga-miR-1612 (target score 97\%). Also, the AKT3 gene has 57 binding sites for 7 miRNA that 21 sites belonged to gga-miR-1612 with target score, 4.1; energy-duplex, -7.82; and $\Delta \mathrm{G}$ open, 6.04 which was the highest score after the FADD gene.

Annealing gga-miR-3536 to 3'-UTR of the PIK3R2 gene with $\Delta \mathrm{G}$ open of 33.56 was the most unstable connection in terms of energy. Of 5 miRNAs identified in the miRDB for this gene, only gga-miR-3536 was identified in miRmap with a score of $85 \%$, because a low score in both programs cannot be selected as a candidate for this gene.

\section{miRNA bind to the TLR pathway}

For the final confirmation of miRNA, the results of miRDB and miRmap were studied simultaneously. For this purpose, miRNAs were confirmed as candidates that both programs received high scores for the target mRNA. Finally, 19 miRNAs for 18 genes of the TLR pathway were identified (Table 4). Since the miRDB scores above 80 were considered significant, so between outputs of miRmap, those miRNAs were selected that received a higher score than other connections.

\section{Discussion}

Inflammation is a natural process to resolve an injury and return the immune system to homeostasis [14, 15]. Recently many studies have focused on understanding the roles of miRNAs in innate immunity [16-18]. Identifying miRNAs involved in controlling the TLR signaling pathways, innate immune responses, and viral 
Table 4. List of final miRNAs predicted binding to TLR pathway

\begin{tabular}{|c|c|c|c|c|}
\hline Gene Symbol & miRNA & Score in miRDB & Score in miRmap & miRNA Sequence and Seed \\
\hline LOC100858824 & gga-miR-16-5p & 87 & 0.39 & $\begin{array}{l}\text { CAAGAACAUCAAACUACCUGAU } \\
\text { TGTTCTT }\end{array}$ \\
\hline PIK3CD & gga-miR-196-1-3p & 94 & 0.48 & $\begin{array}{l}\text { CAAGAACAUCAAACUACCUGAU } \\
\text { TGTTCTT }\end{array}$ \\
\hline РІКЗСВ & gga-miR-214 & 85 & 0.10 & $\begin{array}{l}\text { ACAGCAGGCACAGACAGGCAG } \\
\text { CCTGCTG }\end{array}$ \\
\hline PIK3R5 & gga-miR-6609-3p & 97 & 0.08 & $\begin{array}{l}\text { ACAGCCCCUGUGCUCUGACCGCA } \\
\text { GGGGCTG }\end{array}$ \\
\hline PIK3R1 & gga-miR-6699-5p & 91 & 0.87 & $\begin{array}{l}\text { UGAAGACCUCGUGCAUUGUAGC } \\
\text { GGTCTTC }\end{array}$ \\
\hline АКT3 & gga-miR-1612 & 99 & 4.01 & $\begin{array}{c}\text { GAAGGAAAAGGAGCUGACUGGG } \\
\text { TTTCCTT }\end{array}$ \\
\hline TOLLIP & gga-miR-6706-5p & 83 & 0.64 & $\begin{array}{c}\text { AGGAGAAAUGGAGUGGGCUGAG } \\
\text { T TTCTCC }\end{array}$ \\
\hline MYD88 & gga-miR-7442-5p & 96 & 0.38 & $\begin{array}{l}\text { CACAGGACCAUGGGAGCGUGGC } \\
\text { GTCCTGT }\end{array}$ \\
\hline MAPK10 & gga-miR-6562-5p & 85 & 0.23 & $\begin{array}{c}\text { AGGGGAAAGGAAUGUGCUGUGG } \\
\text { TTTCCCC }\end{array}$ \\
\hline RIPK1 & gga-miR-1687-5p & 83 & 0.26 & $\begin{array}{l}\text { UGAGGAAAUGAGCCAGCUGAG } \\
\text { TT TCCTC }\end{array}$ \\
\hline TBK1 & gga-miR-6633-5p & 81 & 0.61 & $\begin{array}{c}\text { AGAGGAAACUAUGGCUGACUGU } \\
\text { TTTCCTC }\end{array}$ \\
\hline FADD & gga-miR-1612 & 97 & 5.58 & $\begin{array}{c}\text { GAAGGAAAAGGAGCUGACUGGG } \\
\text { TTTCCTT }\end{array}$ \\
\hline TAB2 & gga-miR-1625-3p & 89 & 0.12 & $\begin{array}{l}\text { GCAGCAGAAUAUCCCUGCCAUU } \\
\text { TCTGCTG }\end{array}$ \\
\hline MAР3К7 & gga-miR-9-3p & 90 & 0.24 & $\begin{array}{l}\text { UAAAGCUAGAGAACCGAAUGU } \\
\text { TAGCTTT }\end{array}$ \\
\hline MAPK14 & gga-miR-6639-5p & 82 & 0.21 & $\begin{array}{l}\text { UACAGAACUUCGGCAUGAUUGCU } \\
\text { GTTCTG T }\end{array}$ \\
\hline MAPK13 & gga-miR-1599 & 82 & 0.18 & $\begin{array}{l}\text { GGAGGGAGGAAAAAAAAAAA } \\
\text { CT CCCTC }\end{array}$ \\
\hline MAPK8 & gga-miR-107-3p & 99 & 0.04 & $\begin{array}{c}\text { AGCAGCAUUGUACAGGGCUAUCA } \\
\text { ATGC TGC }\end{array}$ \\
\hline MAPK8 & gga-miR-103-3p & 99 & 0.04 & $\begin{array}{c}\text { AGCAGCAUUGUACAGGGCUAUGA } \\
\text { ATGC TGC }\end{array}$ \\
\hline
\end{tabular}

immune evasion may provide significant clues for detecting novel drug targets in inflammatory and autoimmune diseases, as well as infections [19, 20].

In this regard, the use of computational approaches not only speeds up the process of identifying miRNAs but also reduces the search space to more likely miRNAs that can be experimentally validated by biologists in a lab [14]. Our study presents the first comprehensive view of miRNAs associated with the TLR-signaling pathway in chicken. The results of the analysis identified both genes AKT serine/threonine kinase 3 (AKT3) and Fas Associated via Death Domain (FADD) as the targets for the gga-miR-1612 that received high scores in both miRDB and miRmap programs. 


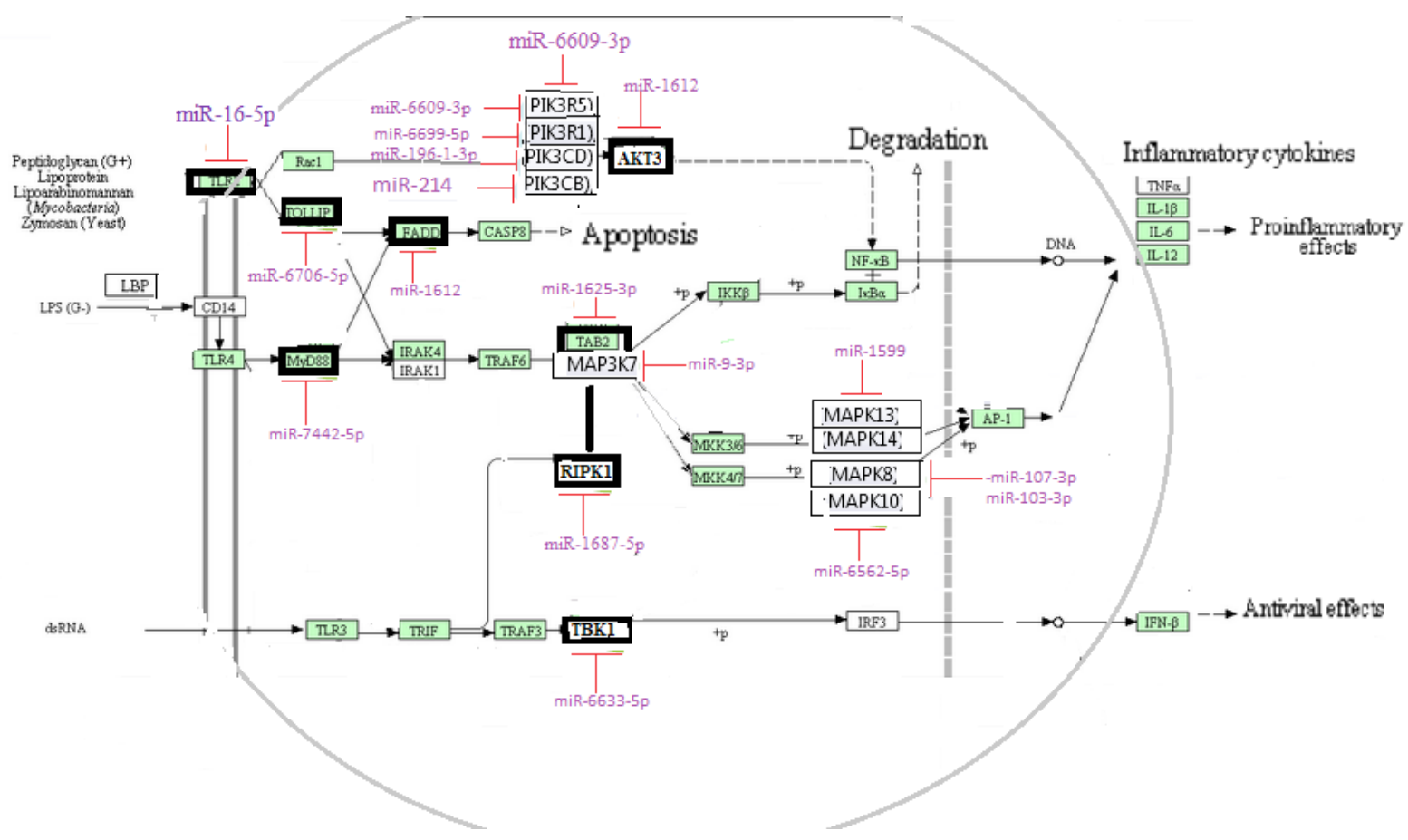

grmm

Figure 1. The fine-tuning of the TLR-signaling pathways of chicken by miRNAs and side effects may occur due to the high expression of these genes

With an in-depth sequencing approach, Wang et al. (2009) identified differentially expressed miRNAs in chicken lung and trachea with avian influenza virus infection [21]. They reported that gga-miR-1612 was upregulated in AIV-infected chicken lungs, while it was explicitly expressed in the tracheae of non-infected ones. They also remarked the different regulation of ggamiR-1612 between lung and trachea and suggested that it might be various mechanisms in response to AIV infection between tissues.

The gga-miR-16-5p and gga-miR-196-1-3p respectively control the LOC100858824 (manufacturer of TLR4) and PIK3CD genes; therefore, they have the same seed (accession number of miRBase; respectively MIMAT0001116 and MIMAT0031017). So, this miRNA with high probability can influence two genes. Phosphatidylinositol-4,5-Bisphosphate 3-Kinase Catalytic Subunit Delta (PIK3CD) is a protein-coding gene that is involved in the immune response.

Li et al. (2017) investigated splenic miRNA expression profiles in host responses to Salmonella Enteritidis infection in chickens [22]. They reported that the relative expression of innate/inflammatory marker genes such as PIK3CD was significantly upregulated following Salmonella Enteritidis infection. The gga-miR-1687-5p influence on 3-UTR of RIPK1 gene as well as gga-miR- 6633-5p influence on TBK1 has the same seed, so the presence of any of these miRNAs increases the possibility of inhibition of another gene.

Overexpression of LOC100858824, TOLLIP, or FADD can lead to apoptosis. Overexpression of MYD88, TAB2, RIPK1, and MAP3K7 leads to degradation, and overexpression of TBK1 leads to the antiviral effects. Also, overexpression of PIK3CD, PIK3CB, PIK3R5, PIK3R1, and AKT3 leads to inflammation effect (Figure 1).

Also, results show that gga-miR-107-3p and gga-miR103-3P can control the position of 379 in the 3'-UTR MAPK8 gene. Li et al. (2017) showed that the expression patterns of gga-miR-103-3p were significantly different between the chicken group with Salmonella Enteritidis infection and non-infected group [22]. Their results showed that LRRC59 is a potential target of ggamiR-103-3p. Although little is known about the function of LRRC59, this family of proteins could have far-reaching effects on the immune response.

A recent study revealed that LRRC59-dependent trafficking of nucleic acid-sensing TLRs might be beneficial for augmentation of antimicrobial immune responses from the endoplasmic reticulum via association with uncoordinated 93 homolog B1. The seed sequence in the 
two miRNAs is similar, but there is a difference in the structure of their hairpin.

Mature gga-miR-103-3p (Acc: MIMAT0001145) is in the position 51 to 73 of hairpin structure, and the guanine base is in position 72, while mature gga-miR-107-3p (Acc: MIMAT0001147) is in position 50 to 72 of hairpin structure and position 71 is cytosine base. Although the bioinformatic methods for the prediction of miRNA are perfectible, it needs subsequent experimental validation. Moreover, as newly identified regulators, the performance mechanism of miRNAs in combination with other regulatory mechanisms will control the outcome of immune responses and these issues should be investigated in future studies.

\section{Ethical Considerations}

\section{Compliance with ethical guidelines}

All ethical principles were considered in this article.

Funding

This work was financialy supported by Department of Agricultural Sciences and Natural Resources, University of Khuzestan, Ahvaz, Iran.

\section{Authors contribution's}

Design, data analysis and performing all of the experiments: Maryam Gholizadeh; Design, supervision, critically revised the manuscript for intellectual content: Jamal Fayazi; Data analysis assist and error checking: Somayeh RahimNahal; All authors reviewed and approved the final version of the manuscript.

\section{Conflict of interest}

The authors declared no conflict of interest.

\section{Acknowledgements}

We express our gratitude to the Department of Agricultural Sciences and Natural Resources, of University of Khuzestan and the anonymous reviewers for their advice for improving paper quality.

\section{References}

[1] Cui J, Gao Y, Chu Q, Bi D, Xu T. miRNA-8159 is involved in TLR signaling pathway regulation after pathogen infection by direct targeting TLR13 in miiuy croaker. Fish Shellfish Immunol. 2017; 66:531-9. [DOI:10.1016/j.fsi.2017.05.046] [PMID]
[2] Dannemann M, Nickel B, Lizano E, Burbano HA, Kelso J. Annotation of primate miRNAs by high throughput sequencing of small RNA libraries. BMC Genomics. 2012; 13:116. [DOI:10.1186/1471-2164-13-116] [PMID] [PMCID]

[3] Anokye-Danso F, Trivedi CM, Juhr D, Gupta M, Cui Z, Tian Y, et al. Highly efficient miRNA-mediated reprogramming of mouse and human somatic cells to pluripotency. Cell Stem Cell. 2011; 8(4):376-88. [DOI:10.1016/j.stem.2011.03.001] [PMID] [PMCID]

[4] Heinrich EM, Dimmeler S. MicroRNAs and stem cells: Control of pluripotency, reprogramming, and lineage commitment. Circ Res. 2012; 110(7):1014-22. [DOI:10.1161/CIRCRESAHA.111.243394] [PMID]

[5] Quinn SR, O'Neill LA. A trio of microRNAs that control Toll-like receptor signalling. Int Immunol. 2011; 23(7):421-5. [DOI:10.1093/intimm/dxr034] [PMID]

[6] Vejnar CE, Blum M, Zdobnov EM. miRmap web: Comprehensive microRNA target prediction online. Nucleic Acids Res. 2013; 41(Web Server issue):W165-8. [DOI:10.1093/nar/gkt430] [PMID] [PMCID]

[7] Baltimore D, Boldin MP, O'Connell RM, Rao DS, Taganov KD. MicroRNAs: New regulators of immune cell development and function. Nat Immunol. 2008; 9(8):839-45. [DOI:10.1038/ ni.f.209] [PMID]

[8] Wang X. miRDB: A microRNA target prediction and functional annotation database with a wiki interface. RNA. 2008, 14(6):1012-7. [DOI:10.1261/rna.965408] [PMID] [PMCID]

[9] Temperley ND, Berlin S, Paton IR, Griffin DK, Burt DW Evolution of the chicken Toll-like receptor gene family: A story of gene gain and gene loss. BMC Genomics. 2008; 9:62. [DOI:10.1186/1471-2164-9-62] [PMID] [PMCID]

[10] Kertesz M, Iovino N, Unnerstall U, Gaul U, Segal E. The role of site accessibility in microRNA target recognition. Nat Genet. 2007; 39(10):1278-84. [DOI:10.1038/ng2135] [PMID]

[11] Chandra V, Girijadevi R, Nair AS, Pillai SS, Pillai RM. MTar: A computational microRNA target prediction architecture for human transcriptome. BMC Bioinformatics. 2010; 11(Suppl 1):S2. [DOI:10.1186/1471-2105-11-S1-S2] [PMID] [PMCID]

[12] Fukui A, Inoue N, Matsumoto M, Nomura M, Yamada K, Matsuda $\mathrm{Y}$, et al. Molecular cloning and functional characterization of chicken toll-like receptors: A single chicken toll covers multiple molecular patterns. J Biol Chem. 2001; 276(50):47143-9. [DOI:10.1074/jbc.M103902200] [PMID]

[13] Gruber AR, Fallmann J, Kratochvill F, Kovarik P, Hofacker IL. AREsite: A database for the comprehensive investigation of AU-rich elements. Nucleic Acids Res. 2011; 39(Database issue):D66-9. [DOI:10.1093/nar/gkq990] [PMID] [PMCID]

[14] Carthew RW, Sontheimer EJ. Origins and Mechanisms of miRNAs and siRNAs. Cell. 2009; 136(4):642-55. [DOI:10.1016/j. cell.2009.01.035] [PMID] [PMCID]

[15] Bandyopadhyay S, Mitra R. TargetMiner: microRNA target prediction with systematic identification of tissue-specific negative examples. Bioinformatics. 2009; 25(20):2625-31. [DOI:10.1093/bioinformatics/btp503] [PMID]

[16] Meunier J, Lemoine F, Soumillon M, Liechti A, Weier M, Guschanski K, et al. Birth and expression evolution of mammalian microRNA genes. Genome Res. 2013; 23(1):34-45. [DOI:10.1101/gr.140269.112] [PMID] [PMCID] 
[17] Jiang L, Shen Y, Zheng K, Li J. Rapid and multiplex microRNA detection on graphically encoded silica suspension array. Biosens Bioelectron. 2014; 61:222-6. [DOI:10.1016/j.bios.2014.05.020] [PMID]

[18] He X, Jing Z, Cheng G. MicroRNAs: New regulators of Tolllike receptor signalling pathways. Biomed Res Int. 2014; 2014:945169. [DOI:10.1155/2014/945169] [PMID] [PMCID]

[19] Berezikov E. Evolution of microRNA diversity and regulation in animals. Nat Rev Genet. 2011; 12(12):846-60. [DOI:10.1038/ nrg3079] [PMID]

[20] Guo H, Ingolia NT, Weissman JS, Bartel DP. Mammalian microRNAs predominantly act to decrease target mRNA levels. Nature. 2010; 466(7308):835-40. [DOI:10.1038/nature09267] [PMID] [PMCID]

[21] Wang Y, Brahmakshatriya V, Zhu H, Lupiani B, Reddy SM, Yoon BJ, et al. Identification of differentially expressed miRNAs in chicken lung and trachea with avian influenza virus infection by a deep sequencing approach. BMC Genomics. 2009; 10:512. [DOI:10.1186/1471-2164-10-512] [PMID] [PMCID]

[22] Li P, Fan W, Li Q, Wang J, Liu R, Everaert N, et al. Splenic microRNA expression profiles and integration analyses involved in host responses to salmonella enteritidis infection in chickens. Front Cell Infect Microbiol. 2017; 7:377. [DOI:10.3389/ fcimb.2017.00377] [PMID] [PMCID] 